\title{
(2) OPEN ACCESS \\ WHO mental health gap action programme (mhGAP) intervention guide: updated systematic review on evidence and impact
}

\author{
Roxanne Keynejad ำ ,' Jessica Spagnolo, ${ }^{2,3}$ Graham Thornicroft ${ }^{1}$
}

- Additional supplemental material is published online only. To view, please visit the journal online (http://dx.doi. org/10.1136/ebmental-2021300254)

1 Department of Health Service and Population Research, Institute of Psychiatry, Psychology and Neuroscience, King's College London, London, UK

2Département des Sciences de la Santé Communautaire, Université de Sherbrooke, Sherbrooke, Quebec, Canada ${ }^{3}$ Centre de recherche Charles-Le Moyne - Saguenay-Lac-SaintJean sur les innovations en santé, Campus de Longueuil, Université de Sherbrooke, Sherbrooke, Quebec, Canada

\section{Correspondence to} Dr Roxanne Keynejad, Department of Health Service and Population Research, Institute of Psychiatry, Psychology and Neuroscience, King's College London, London SE5 8AF, UK; Roxanne.1. keynejad@kcl.ac.uk

Received 7 February 2021 Revised 22 March 2021 Accepted 29 March 2021 Published Online First 26 April 2021

\section{Check for updates}

(c) Author(s) (or their employer(s)) 2021. Re-use permitted under CC BY. Published by BMJ.

To cite: Keynejad $R$, Spagnolo J, Thornicroft G Evid Based Ment Health 2021:24:124-130.

\section{ABSTRACT}

Question There is a large worldwide gap between the service need and provision for mental, neurological and substance use disorders. WHO's Mental Health Gap Action Programme (mhGAP) intervention guide (IG), provides evidence-based guidance and tools for assessment and integrated management of priority disorders. Our 2017 systematic review identified 33 peerreviewed studies describing mhGAP-IG implementation in low-income and middle-income countries.

Study selection and analysis We searched MEDLINE, Embase, PsycINFO, Web of Knowledge, Scopus, CINAHL, LILACS, ScieELO, Cochrane, PubMed databases, 3ie, Google Scholar and citations of our review, on 24 November 2020. We sought evidence, experience and evaluations of the mhGAP-IG, app or mhGAP Humanitarian IG, from any country, in any language. We extracted data from included papers, but heterogeneity prevented meta-analysis.

Findings Of 2621 results, 162 new papers reported applications of the mhGAP-IG. They described mhGAP training courses (59 references), clinical applications $(n=49)$, research uses $(n=27)$, contextual adaptations $(n=13)$, economic studies $(n=7)$ and other educational applications $(n=7)$. Most were conducted in the African region (40\%) and South-East Asia (25\%). Studies demonstrated improved knowledge, attitudes and confidence post-training and improved symptoms and engagement with care, post-implementation. Research studies compared mhGAP-IG-enhanced usual care with task-shared psychological interventions and adaptation studies optimised mhGAP-IG implementation for different contexts. Economic studies calculated human resource requirements of scaling up mhGAP-IG implementation and other educational studies explored its potential for repurposing.

Conclusions The diverse, expanding global mhGAP-IG literature demonstrates substantial impact on training, patient care, research and practice. Priorities for future research should be less-studied regions, severe mental illness and contextual adaptation of brief psychological interventions.

\section{BACKGROUND}

There is a well-reported, very sizeable global gap between the need and provision of services to prevent, identify and treat mental, neurological and substance use (MNS) disorders. To expedite care as efficiently as possible, the WHO's World Mental Health Report ${ }^{1}$ recommended the assessment and management of MNS disorders in primary healthcare (PHC) and community settings, as outlined in the Mental Health Action Plan. ${ }^{2}$ To deliver community mental healthcare, PHC staff require training in the assessment, diagnosis and management of MNS disorders, alongside other key components, including regular supervision and support. Central to this agenda is the WHO Mental Health Gap Action Programme ${ }^{3}$ (mhGAP) and intervention guide (IG). ${ }^{4}$

The mhGAP-IG is an evidence-based tool for the assessment and treatment of priority MNS disorders, featuring guidelines for clinical decision making. Aimed at non-specialist PHC staff in lowincome and middle-income countries (LMICs), the mhGAP-IG is also used by governments, nongovernmental organisations and researchers. An mhGAP Humanitarian Intervention Guide (HIG), ${ }^{5}$ for settings characterised by widespread psychological trauma and even less access to mental health specialists, has also been published, ${ }^{5}$ alongside an mhGAP-IG mobile device app. ${ }^{6}$

Our previous systematic review ${ }^{7}$ identified 33 published peer-reviewed studies and protocols which used the mhGAP-IG. Given that another review found only six published studies of experimental non-communicable disease clinical guideline implementation in $\mathrm{LMICs},{ }^{8}$ the mhGAP-IG literature was relatively substantial in 2017. The mhGAP-IG had been adopted by clinicians, government ministries, trainers, educators and academics in diverse LMICs. Applications ranged from local adaptation, training and clinical practice, to mobile applications for isolated PHC staff, tablet-based avatar-assisted family training, economic modelling, novel rating scales and comparison interventions in randomised controlled trials (RCTs). The literature was, however, dominated by studies from a subset of countries, suggesting that much implementation was not evaluated, or that evaluations were not widely disseminated. Reliance on relatively limited pre-mhGAP and post-mhGAP training knowledge assessments meant that opportunities may have been missed at times, to describe real-world contextual challenges to widespread uptake and scale-up.

\section{OBJECTIVE}

In this study, we sought to identify evidence generated since the publication of our previous review for the practical implementation of the WHO mhGAP-IG and associated tools, in terms of how they have been used, evaluated and reported. 

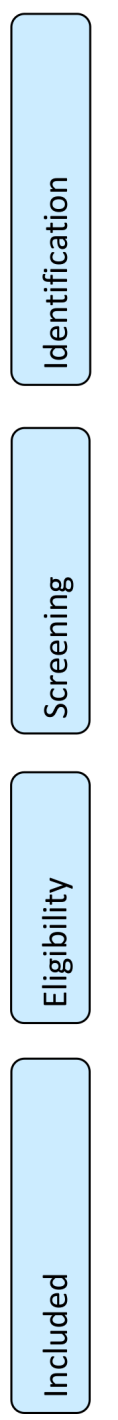

Records identified through database searching $(n=2620)$
Additional records identified through other sources $(n=1)$

59 records of mhGAP-IG training courses (58 studies)

49 records of clinical uses of mhGAP-IG (46 studies)

27 records of research studies using the mhGAP-IG (25 studies)

13 records of contextual adaptations of mhGAP-IG (12 studies)

7 records of economic analysis using mhGAP-IG

7 records of other educational applications of the mhGAP-IG

Figure 1 PRISMA flow diagram. mhGAP-IG, Mental Health Gap Action Programme Intervention Guide; PRISMA, Preferred Reporting Items for Systematic Reviews and Meta-Analyses.

\section{STUDY SELECTION AND ANALYSIS}

We included any type of study design, review or report of evidence, experience or evaluation of using the mhGAP-IG in LMICs, from any country, in any language. In order to identify as many potentially relevant records as possible, we searched the following databases on 24 November 2020: 3ie, Cochrane Library, CINAHL, EMBASE, LILACS, Medline, PsycINFO, PubMed, SciELO, Scopus, Web of Knowledge. The only search terms used were 'mental health gap action programme' OR 'mental health gap action program' OR 'mhGAP'. The term 'intervention guide' was not included, due to its variable use in the literature and in practice. Searches were conducted in English, but studies written in other languages were eligible for inclusion. In addition to database searches, the reference lists of relevant excluded papers were searched for eligible studies. Grey literature, including book chapters and conference presentations, were identified by repeating the search on Google Scholar. We used forward citation tracking of our 2017 systematic review to identify additional records. ${ }^{7}$ This work is registered on the International Prospective Register of Systematic Reviews (registration number: CRD42017068459).

\section{FINDINGS}

Figure 1 shows the flow of studies from identification to screening, eligibility and inclusion. The titles and abstracts of the 2002 non-duplicated papers were screened by RK excluding 1807, which did not review or report evidence, experience or evaluation the mhGAP-IG, app or HIG. The details of a subset of excluded studies reporting non-mhGAP-IG-related integrations of mental healthcare into PHC were recorded for a separate review. The remaining 195 full-text articles were screened again for eligibility by JS and categorised into study types. Where studies could be categorised into more than one type (e.g., both clinical application and research), a judgement was made about the study's primary focus. No papers were excluded based on language, and no relevant papers from high-income settings were identified.

Table 1 shows the distribution of study types across geographical regions. Most studies $(40 \%, n=62)$ were conducted in the African region and South-East Asia $(25 \%, \mathrm{n}=38)$. Notably few studies were conducted in the Eastern Mediterranean $(7 \%$, $\mathrm{n}=10)$, region of the Americas $(4 \%, \mathrm{n}=6)$ and the European region $(1 \%, n=2)$. Ten per cent of studies $(n=15)$ reported results from at least two countries on different continents. Most 
Table 1 Studies reporting mhGAP-IG use, experience or evaluation, by type and geographical region

\begin{tabular}{|c|c|c|c|c|c|c|c|}
\hline Types $\rightarrow$ & & & & & & & \\
\hline Regions $\downarrow$ & Training courses & Clinical uses & Research & Contextual adaptation & Economic analysis & Other educational & Total studies \\
\hline African Region & 23 & 25 & 8 & 4 & 2 & 0 & 62 \\
\hline South-East Asia & 10 & 13 & 9 & 3 & 0 & 3 & 38 \\
\hline Multicontinental & 4 & 4 & 1 & 3 & 3 & 0 & 15 \\
\hline Western Pacific & 7 & 1 & 2 & 0 & 1 & 1 & 12 \\
\hline Region of the Americas & 5 & 1 & 0 & 0 & 0 & 0 & 6 \\
\hline Eastern Mediterranean & 8 & 2 & 5 & 1 & 1 & 0 & 10 \\
\hline European Region & 1 & 0 & 0 & 0 & 0 & 1 & 2 \\
\hline Other & 0 & 0 & 0 & 1 & 0 & 2 & 3 \\
\hline Total & 58 & 46 & 25 & 12 & 7 & 7 & 155 \\
\hline
\end{tabular}

mhGAP-IG, Mental Health Gap Action Programme Intervention Guide.

included studies described uses of the mhGAP-IG, app or HIG for training $(38 \%, \mathrm{n}=58)$, clinical use $(30 \%, \mathrm{n}=44)$ and research $(16 \%, \mathrm{n}=25)$.

Table 2 shows the study design methods used across different study types. RCTs comprised 15\% $(n=23)$ of studies and RCT protocols or trial registrations, $12 \%(n=19)$. Uncontrolled studies comprised $23 \%$ of studies $(n=36)$, descriptive accounts $18 \%$ of studies $(n=28)$, qualitative studies $8 \%(n=13)$ and other non-randomised designs $7 \%(n=10)$.

Of the 193 eligible studies, data were extracted by JS and RK from the 162 papers (describing 155 studies) not included in the 2017 systematic review $(n=33)$. The heterogeneity of mhGAP-IG, app and HIG uses, outcome measures, and evaluations precluded meta-analysis. Due to the heterogeneity of designs, including a large proportion of non-randomised designs and variable reporting methods, we did not assess risk of bias across included studies. ${ }^{9}$ Extracted data included country involved, participants, sample, nature of use, evaluation conducted and summary of findings, where relevant. Below, we summarise the findings reported by studies categorised into six different types: use in training (online supplemental material table 4), use in clinical practice (online supplemental material table 5), use in research (online supplemental material table 6), local adaptation or contextualisation (online supplemental material table 7), economic evaluation (online supplemental material table 8 ) and other educational purposes (online supplemental material table 9).

\section{Use in training}

Of the 162 included papers, 58 studies (59 references) reported the use of the mhGAP-IG or HIG in training courses, trained PHC and other community-based staff (health extension workers, community health workers (CHWs), nurses, midwives, pharmacists, physician assistants, clinical officers, general practitioners, social workers), students (nursing and medical undergraduates, master's students), research assistants, clinical educators, decision-makers, caregivers, traditional and faith healers, and mental health service users.

The impact of mhGAP-IG-based training was evaluated using cross-sectional designs, cohort studies, pretest/post-test studies, retrospective medical records reviews and RCTs. Overall, postmhGAP-IG training, PHC and community-based staff showed increased mental health knowledge and awareness, improved attitudes towards mental ill-health and people living with mental health problems, improved attitudes towards psychiatry, more confidence in managing mental health problems in PHC, increased job satisfaction and interest in mental health training. Evaluations of mhGAP-IG-based training highlighted trainees' satisfaction with the programme, which was generally considered useful, relevant and valuable.

Aspects of trainees' practice also improved. Studies reported improved diagnostic skills and diagnostic agreement, increased mental health service efficiency, and greater family involvement in care. Studies also reported benefits for patients following mhGAP-IG-based staff training. These included increased mental

Table 2 Methods used in each category of study design

\begin{tabular}{|c|c|c|c|c|c|c|c|}
\hline Types $\rightarrow$ & & & & & & & \\
\hline Methods $\downarrow$ & Training courses & Clinical uses & Research & Contextual adaptation & Economic analysis & Other educational & Total studies \\
\hline $\mathrm{RCT}$ & 4 & 8 & 10 & 0 & 0 & 1 & 23 \\
\hline RCT protocol/trial registration & 3 & 10 & 6 & 0 & 0 & 0 & 19 \\
\hline Uncontrolled study & 22 & 12 & 0 & 0 & 1 & 1 & 36 \\
\hline Descriptive account & 16 & 6 & 1 & 5 & 0 & 0 & 28 \\
\hline Qualitative study & 5 & 1 & 0 & 6 & 0 & 1 & 13 \\
\hline Other non-randomised design & 4 & 4 & 1 & 0 & 1 & 0 & 10 \\
\hline Other protocol & 1 & 3 & 3 & 0 & 0 & 0 & 7 \\
\hline Feasibility study & 3 & 2 & 2 & 0 & 0 & 0 & 7 \\
\hline Economic study & 0 & 0 & 0 & 0 & 5 & 0 & 5 \\
\hline Non-clinical study & 0 & 0 & 2 & 1 & 0 & 4 & 5 \\
\hline Total & 58 & 46 & 25 & 12 & 7 & 7 & 155 \\
\hline
\end{tabular}

$\mathrm{RCT}$, randomised controlled trial. 
health service utilisation, clinical improvement, returning to work, fewer discriminatory experiences and greater satisfaction with services.

Descriptive accounts and qualitative studies of mhGAPIG-based training highlighted barriers to training PHC and community-based staff. These included limited funding or institutional support for training, difficulty establishing supervision mechanisms, cultural differences in conceptualisations of mental ill-health, the need for further training and time constraints. Descriptive accounts and qualitative studies also emphasised the importance of continued supervision, medication supplies, coordination of services, political commitment to mental healthcare, planning and leadership to the success of mhGAP-IG-based training.

Four records describing training courses were protocols or trial registrations, from Iran, Sri Lanka India and Nepal. These studies aimed to use mhGAP-IG-based training to improve youth and parent functioning, reduce mental health symptoms, improve clinical skills and reduce stigma among health workers.

\section{Use in clinical practice}

Forty-six studies (49 references) reported clinical outcomes of applications of the mhGAP-IG or HIG in practice(online supplemental material table 5). Target patient groups included adults with mood or anxiety disorders, adults with severe mental illness (SMI), pregnant women with mood disorders, parents of primary school-aged children and people living with HIV.

Studies assessed the mhGAP-IG and HIG's impact in clinical practice through retrospective medical records reviews, uncontrolled studies, cross-sectional studies, case-control studies and RCTs. They reported positive impacts of the mhGAP-IG on mental health awareness, clinician-confirmed case identification, mental health symptoms, clinical recovery, experiences of discrimination, food security, quality of life, contact coverage and access to care, for example, clinical consultation numbers, intervention adherence, retention in treatment and numbers of facilities treating chronic illness. Studies explored factors influencing the mhGAP-IG and HIG's clinical uptake. These included confidential consultation space, patient trust in health workers, mental health stigma, medication supply, staff (including psychiatrist) availability, staff turnover, supervision, support and health worker motivation.

Some studies used RCT designs to evaluate clinical use of the mhGAP-IG alone, while others combined the mhGAP-IG with problem-solving therapy, behavioural activation, interpersonal therapy, parent skills training, communication skills and counselling. In some cases, the mhGAP-IG was provided to both intervention and control groups as enhanced usual care.

Ten records evaluating clinical use of the mhGAP-IG, app or HIG were protocols and three were trial registrations, from Nigeria, Ghana, Kenya, Uganda Rwanda, South Africa, Ethiopia, India, Nepal and Pakistan. These studies aimed to improve patient symptoms or functioning, parent-child communication and interaction, parent quality of life and the feasibility of combining mhGAP-IG-informed care with interpersonal therapy. One trial registration will compare the electronic mhGAP-IG with the paper version.

\section{Use in research}

Most of the studies included in this review employed research methods in some form. We categorised 25 studies $(27$ references) as reporting uses of the mhGAP-IG for research where the primary purpose of the work was a research endeavour, whose conduct was facilitated by the mhGAP-IG as a tool(online supplemental material table 6). Ten studies (seven RCTs, two feasibility studies and one non-randomised trial) used the mhGAP-IG to standardise the mental healthcare received by participants in both intervention and control arms. In India, Counselling for Alcohol Problems was associated with significantly more remission and abstinence from alcohol use disorder at 3 and 12 months' follow-up than mhGAP-IG-enhanced usual care. Also in India, the Healthy Activity Programme behavioural activation intervention was associated with significantly lower depressive symptoms and increased remission at 3 and 12 months' follow-up than mhGAP-IG-enhanced usual care. In Pakistan, there was no difference between the impact of mhGAPIG-enhanced usual care and an intensive group-based psychosocial intervention on depressive symptoms and remission at 6 and 36 months' follow-up. Also in Pakistan, there was no difference between electronic health records and decision support for integrated management of chronic conditions (including depression, using mhGAP-IG content) and non-electronic training. In Nepal, a feasibility study found that group problem management plus $(\mathrm{PM}+)$ was acceptable and feasible to compare with mhGAPIG-enhanced usual care. In Somaliland, a non-randomised study found that mhGAP-IG-informed community-based relapse prevention was more effective for patients commencing the programme in remission than those requiring intensive homebased care first.

Two RCTs used the mhGAP-IG to inform the content of a novel intervention in Pakistan and China and one used it to screen for psychosis and assess suicide risk during a trial in Malaysia. In Pakistan, more pregnant women sought help for distress from CHWs following mhGAP-IG-informed psychoeducation than women in the control arm. In China, an mhGAP-IGinformed intervention was associated with greater reductions in anticipated discrimination, negative symptoms and functioning scores than a community psychiatrist interview. In Malaysia, common mental disorder symptoms improved more in participants receiving integrative adapt therapy than those receiving cognitive behavioural therapy.

One validation study used the mhGAP-IG to provide treatment for adolescents diagnosed with a mental disorder using a newly adapted screening tool in Ethiopia. The adapted Amharic Youth Self Report was a reliable and valid screen for anxiety, depression and social problems in female adolescents and attention problems in young men. One study developed a scale to categorise free Arabic-language anxiety and depression apps using the mhGAP-IG. One descriptive account incorporated the mhGAP-IG into a novel counselling aid (FELICIA) based on the Thinking Healthy Programme, for couples experiencing infertility in Nigeria; a pilot study is underway.

Nine studies using the mhGAP-IG for research were protocols: six RCTs, a feasibility study, an implementation study and a cohort study. One prospective cohort study will evaluate the impact on tuberculosis outcomes of mhGAP-IG-based depression treatment. Five RCTs will use the mhGAP-IG to standardise the mental healthcare received by participants in both intervention and control arms, in Ethiopia, Tanzania, Pakistan and Nepal. A sixth RCT protocol aims to compare the reach, costs and clinical effectiveness of mhGAP-IG-based care delivered by specialist mental health professionals, PHC staff and CHWs in Mozambique. The implementation study protocol aims to evaluate implementation strategies for cognitive stimulation therapy in Brazil, India and Tanzania. 
Table 3 Facilitators and barriers to mhGAP-IG implementation success identified by qualitative studies

\begin{tabular}{|c|c|c|}
\hline System level & Facilitators & Barriers \\
\hline Service user & $\begin{array}{l}\text { Ability to see traditional healers alongside biomedical care. }{ }^{31} \\
\text { Community awareness raising. }{ }^{32}\end{array}$ & $\begin{array}{l}\text { Cultural differences with the biomedical model. }{ }^{33} \\
\text { Different perspectives on appropriate treatment. }{ }^{34-36} \\
\text { Rural residence, distance from health facilities, thinking that problems will self-resolve, } \\
\text { concerns about treatment costs. }{ }^{37} \\
\text { Resistance to treatment. }{ }^{38}\end{array}$ \\
\hline Staff & $\begin{array}{l}\text { Health worker motivation. }{ }^{39} \\
\text { Sharing research findings collaboratively. }{ }^{40}\end{array}$ & $\begin{array}{l}\text { Resistance from faculty members. }{ }^{41} \\
\text { Time constraints and workload. }{ }^{42} 43 \\
\text { Stigma. }{ }^{38} 3943 \\
\text { Mistrust of informal health providers, cultural misunderstandings. }{ }^{38}\end{array}$ \\
\hline Service & $\begin{array}{l}\text { Supervision. }{ }^{39} 4244 \\
\text { Onward referral. } \\
\text { Reliable psychotropic medicine procurement. }{ }^{32} 3944 \\
\text { Trained female community health volunteers. }{ }^{32}\end{array}$ & $\begin{array}{l}\text { Scarcity of specialist staff to deliver supervision. }{ }^{39} 45 \\
\text { Lack of financial resources } s^{3841} \\
\text { Limited referral systems. }{ }^{38} \\
\text { Staff turnover, lack of confidential space for consultation. }{ }^{39}\end{array}$ \\
\hline Leadership & Strong political and organisational support. ${ }^{35} 363940$ & Lack of institutional support. ${ }^{41}$ \\
\hline
\end{tabular}

\section{Local adaptation or contextualisation}

Twelve studies (13 references) reported local adaptation or contextualisation of the mhGAP-IG(online supplemental material table 7). Qualitative studies, case studies and descriptive accounts included reflections and experiences of adapting or contextualising the mhGAP-IG. Methods included ethnographic research, in-depth stakeholder interviews and focus groups, theory of change workshops, literature reviews, local epidemiological evidence reviews, situational analyses and asset mapping of community resources.

Table 3 summarises facilitators and barriers to mhGAP-IG implementation identified by qualitative studies, at service user, staff, service and leadership levels. Consistent themes included integrating the biomedical model with cultural perspectives, addressing stigma, adjusting staff workloads, providing supervision, creating referral pathways and obtaining institutional and political support.

Recommended considerations when adapting or contextualising the mhGAP-IG included cultural differences in explanatory models and attitudes towards mental disorders, the structure of the local health system, the availability of supervision and support post-training, trainees' prior education, knowledge and skills, trainee recruitment processes and the wider sociopolitical context. ${ }^{10}$ Authors also recommended considering the availability of specialists for referral, medication supplies, translation of the IG into local languages and organisational roles. ${ }^{11} 12$

One study developed a framework to integrate cultural knowledge, structural competence and ethics into mhGAP-IG planning, adaptation, training and implementation. Domains included examining concepts of wellness and illness, exploring systems of care, recognising formal and informal care systems, and considering the ethical space of power dynamics and decision making. ${ }^{13}$

\section{Economic evaluation}

Seven studies (seven references) used the mhGAP-IG to conduct economic evaluations(online supplemental material table 8). Three studies used the mhGAP costing tool to calculate human resource requirements from the prevalence of depression in Syria, MNS disorders in Pacific Island and 48 sub-Saharan African countries. Two studies performed cost-benefit analyses of the scale-up of effective treatments for depression and anxiety in 36 countries, and for psychosis, depression and epilepsy in Ethiopia, India, Nepal and Uganda. Two studies assessed household out-of-pocket health expenditures, finding that having a relative with SMI was associated with catastrophic health expenditure, financial dissatisfaction and cost-cutting actions, such as withdrawing children from school. Functional impairment was associated with higher treatment costs in Ethiopia and India, and out-of-pocket expenditure in Uganda and India.

\section{Other educational purposes}

The final seven studies reported uses of the mhGAP-IG for a range of healthcare education-focused purposes(online supplemental material table 9). Two described the development of a community informant detection tool in Nepal based on mhGAP-IG modules and its cluster RCT evaluation, which found that it significantly increased diagnoses. One study developed an mhGAP-IG-based electronic decision support system in India, where it was acceptable but required modification. An Indonesian study used the mhGAP-IG to code interview responses, identifying greater mental health knowledge and lower stigma among specialist health workers, compared with non-specialists. In Ukraine, the incorporation of the mhGAP-IG into the undergraduate medical curriculum was associated with sustainability, cost-effectiveness, educational and care quality benefits. One study designed mhGAP-IG-based clinician competencies to inform training and assessment. A qualitative study used participant observations of the mhGAP-IG's use to analyse and critique its algorithmic approach.

\section{CONCLUSIONS AND CLINICAL IMPLICATIONS}

This updated systematic review of the literature on the mhGAP-IG, app and HIG demonstrates a dramatic increase in published studies of its use and evaluation since $2017 .^{7}$ Most of the literature comes from the African region and South-East Asia, with notable evidence gaps including the region of the Americas and the Eastern Mediterranean. Countries participating in the programme for improving mental healthcare (PRIME) study (Ethiopia, India, Nepal, South Africa and Uganda) are well represented, demonstrating the benefits to mental health research and evaluation of investment, shared training and methodologies. PRIME identified barriers to integrating mental health into PHC, including limited funding, insufficient specialists to supervise non-specialist workers, inadequate health system structures to support roll-out of task-shared interventions, low community awareness of mental health and high levels of stigma. ${ }^{14}$ Building on PRIME, the AFFIRM study is conducting two RCTs in South Africa and Ethiopia of task-shared psychological interventions, building capacity through fellowships and mentorships for early career researchers from Ethiopia, Ghana, Malawi, Uganda and 
Zimbabwe, and building a network for interdisciplinary collaboration. ${ }^{15}$ Both projects demonstrate ways of enhancing the impact and sustainability of mental health research, through long-term collaborations between LMIC and HIC institutions, funded to design and evaluate new interventions from piloting to scale-up, and develop the next generation of global mental health researchers in LMICs.

Similar study types to those identified in our 2017 review $^{7}$ have been published since, with the highest proportions of studies reporting on mhGAP-IG training courses, clinical and research applications. The surge in literature also includes contextual adaptations, economic analyses and novel applications to health education. Studies demonstrated the benefits of the mhGAP-IG's use in training for improving the knowledge, attitudes and confidence of health workers in relation to mental health disorders. Clinical studies identified improvements in patient symptoms, quality of life, access to and engagement with mental healthcare associated with mhGAP-IG implementation.

The published evidence comprises a breadth of research methods, with high proportions of RCTs and protocols for RCTs, and smaller proportions of related feasibility studies, implementation studies and process evaluations. The publication of uncontrolled, non-randomised, descriptive and case studies contributes a valuable literature on the mhGAP-IG from settings where more extensive research studies may not be feasible. Adaptation studies recommended a range of contextual considerations to optimise the success of mhGAP-IG implementation.

Many research uses of the mhGAP-IG aimed to adapt and evaluate brief psychological interventions for settings where specialist mental healthcare is limited or unavailable. Often, such interventions were implemented alongside mhGAP-IG training (as 'enhanced usual care') or as a next step after successful mhGAP-IG training for clinical use. Literature on the Thinking Healthy Programme, Healthy Activity Programme, Counselling for Alcohol Problems and PM+, largely from South Asia, demonstrates the broader role of the mhGAP-IG in encouraging the development and rigorous evaluation of acceptable, feasible and scalable talking therapies in LMICs. Sharing of intervention manuals via the WHO website should facilitate the adaptation and evaluation of these interventions for other countries, cultures and contexts. SMIs such as schizophrenia, severe depression and bipolar affective disorder in PHC were less frequently targeted by intervention studies, the majority of which focused on mild to moderate depression. Lessons learned from the use of the mhGAP-IG for common mental disorder research could now be applied to research and intervention development for people with SMI.

Recognition has grown of the need to improve the quality of inpatient and community mental healthcare, attend to the human rights of people with mental disorders and promote recovery. ${ }^{16}$ The fact that most mhGAP-IG, app and HIG training combined lectures and small-group tasks with role play interactions suggests that scaling up the mhGAP-IG could have wider benefits, improving health worker communication skills and the compassion and respect with which care is delivered. Twenty-one included studies addressed or measured stigma, or planned to. Of 15 completed studies, nine reported reductions in one or more measures of stigma, even where health worker knowledge or skills were unchanged. ${ }^{17-25}$ Economic studies identified the scale of the mental health gap, its financial ${ }^{26}$ and disability impacts, ${ }^{27}$ and calculated the workforce requirements ${ }^{28-30}$ and costs of treatment.

The principal limitation of this study is the likelihood of publication bias, with studies finding no significant or negative results less likely to have been published. A substantial number of reports is likely to remain in the 'grey literature' outside peer reviewed journals. A further limitation is that we conducted single-author screening of potentially eligible studies, raising the risk that some eligible studies could have been missed.

Since their release, the WHO mhGAP-IG, app and HIG have made a remarkable impact on global mental health education and training, clinical practice, research and policy. Included studies used a range of methods: a testament to practitioners' commitment to mhGAP-IG monitoring, evaluation and implementation research. Promising approaches to strengthen the evidence base include consortia bringing researchers together from a range of LMICs and HICs for collaborative, interdisciplinary study, benefiting from shared learning and pooled resources. The mhGAP-IG has created a dynamic field in which practitioners from diverse international contexts learn from each other's experiences and adapt study designs and intervention models for their own context. The next step must be to fill gaps in the evidence base for under-studied regions and disorders, and to investigate the scale-up and sustainable integration of isolated interventions into long-term clinical practice.

\section{Twitter Roxanne Keynejad @RoxanneKeynejad}

Contributors RK devised the study strategy, registered the study, conducted the search, extracted data, led the write-up, editing and submission. JS reviewed potentially eligible studies, extracted data, wrote half of the findings section, edited and approved the manuscript. GT initiated the study, supervised the study, edited and approved the manuscript.

Funding This work was funded by the WHO. RK is supported by a King's IoPPN Clinician Investigator Scholarship for her PhD and by the National Institute of Health Research (NIHR) Global Health Research Unit on Health System Strengthening in sub-Saharan Africa (ASSET), King's College London (GHRU 16/136/54) using UK aid from the UK government. JS is supported by a postdoctoral training award, funded by Fonds de recherche santé - Québec (\#284461). GT is supported by the National Institute for Health Research (NIHR) Applied Research Collaboration South London at King's College London NHS Foundation Trust, and by the NIHR Asset Global Health Unit award. The views expressed are those of the author(s) and not necessarily those of the NHS, the NIHR or the Department of Health and Social Care. GT also receives support from the National Institute of Mental Health of the National Institutes of Health under award number R01MH100470 (Cobalt study). GT is supported by the UK Medical Research Council in relation the Emilia (MR/S001255/1) and Indigo Partnership (MR/R023697/1) awards.

Disclaimer The authors alone are responsible for the views expressed in this publication and they do not necessarily represent the decisions, policy or views of the WHO.The views expressed in this publication are those of the authors and not necessarily those of the NIHR or the Department of Health and Social Care.

Competing interests GT chaired the Guideline Development Group for the WHO mhGAP Intervention Guide.

Patient consent for publication Not required.

Provenance and peer review Not commissioned; externally peer reviewed.

Data availability statement Data sharing is not applicable as no datasets were generated and/or analysed for this study. The data analysed in this systematic review came from publications of research studies whose data may be sought by direct contact with the study authors.

Supplemental material This content has been supplied by the author(s). It has not been vetted by BMJ Publishing Group Limited (BMJ) and may not have been peer-reviewed. Any opinions or recommendations discussed are solely those of the author(s) and are not endorsed by BMJ. BMJ disclaims all liability and responsibility arising from any reliance placed on the content. Where the content includes any translated material, BMJ does not warrant the accuracy and reliability of the translations (including but not limited to local regulations, clinical guidelines, terminology, drug names and drug dosages), and is not responsible for any error and/or omissions arising from translation and adaptation or otherwise.

Open access This is an open access article distributed in accordance with the Creative Commons Attribution 4.0 Unported (CC BY 4.0) license, which permits others to copy, redistribute, remix, transform and build upon this work for any purpose, provided the original work is properly cited, a link to the licence is given, 
and indication of whether changes were made. See: https://creativecommons.org/ licenses/by/4.0/.

\section{ORCID iD}

Roxanne Keynejad http://orcid.org/0000-0003-4434-3526

\section{REFERENCES}

1 WHO. The world health report 2001: mental health: new understanding, new hope. World Health Organization, 2001.

2 WHO. Mental health action plan 2013-2020, 2013. Available: https://www.who.int/ publications/i/item/9789241506021

3 WHO. Who mental health gap action programme (mhGAP), 2020. Available: https:// www.who.int/mental_health/mhgap/en/

4 WHO. mhGAP Intervention Guide - Version 2.0, 2016. Available: https://www.who.int/ publications//item/mhgap-intervention-guide-version-2.0

5 WHO. mhGAP humanitarian intervention guide (mhGAP-HIG), 2015. Available: https://www.who.int/mental_health/publications/mhgap_hig/en/

6 WHO. Who releases mhGAP intervention guide APP, 2017. Available: https://www. who.int/mental_health/mhgap/e_mhgap/en/

7 Keynejad RC, Dua T, Barbui C, et al. Who mental health gap action programme (mhGAP) intervention guide: a systematic review of evidence from low and middleincome countries. Evid Based Ment Health 2018:21:30-4.

8 Docherty M, Shaw K, Goulding L, et al. Evidence-Based guideline implementation in low and middle income countries: lessons for mental health care. Int J Ment Health Syst 2017;11:8.

9 Higgins JPT, Altman DG, Sterne JAC. Assessing risk of bias in included studies. In: Cochrane Handbook for systematic reviews of interventions. The Cochrane Collaboration, 2011.

10 Faregh N, Lencucha R, Ventevogel P, et al. Considering culture, context and community in mhGAP implementation and training: challenges and recommendations from the field. Int J Ment Health Syst 2019;13:58.

11 Doherty S, Dass G, Edward A, et al. Challenges and lessons learned in re-filming the WHO mhGAP training videos for Sri Lankan context - a qualitative study. Confl Health 2020;14:7.

12 Bitta MA, Kariuki SM, Omar A, et al. Contextualizing and pilot testing the mental health gap action programme intervention guide (mhGAP-IG) to primary healthcare workers in Kilifi, Kenya. Glob Ment Health 2020;7:e11.

13 Gómez-Carrillo A, Lencucha R, Faregh N, et al. Engaging culture and context in mhGAP implementation: fostering reflexive deliberation in practice. BMJ Glob Health 2020;5.

14 Davies T, Lund C. Integrating mental health care into primary care systems in lowand middle-income countries: lessons from prime and affirm. Glob Ment Health 2017;4:e7.

15 Lund C, Alem A, Schneider M, et al. Generating evidence to narrow the treatment gap for mental disorders in sub-Saharan Africa: rationale, overview and methods of affirm. Epidemiol Psychiatr Sci 2015;24:233-40.

16 WHO. Who QualityRights initiative - improving quality, promoting human rights, 2019. Available: https://www.who.int/mental_health/policy/quality_rights/en/

17 Kohrt BA, Mutamba BB, Luitel NP, et al. How competent are non-specialists trained to integrate mental health services in primary care? global health perspectives from Uganda, Liberia, and Nepal. Int Rev Psychiatry 2018:30:182-98.

18 Kohrt BA, Turner EL, Rai S, et al. Reducing mental illness stigma in healthcare settings: proof of concept for a social contact intervention to address what matters most for primary care providers. Soc Sci Med 2020;250:112852.

19 Li J, Fan Y, Zhong H-Q, et al. Effectiveness of an anti-stigma training on improving attitudes and decreasing discrimination towards people with mental disorders among care assistant workers in Guangzhou, China. Int J Ment Health Syst 2019;13:1.

20 Li J, Huang Y-G, Ran M-S, et al. Community-Based comprehensive intervention for people with schizophrenia in Guangzhou, China: effects on clinical symptoms, social functioning, internalized stigma and discrimination. Asian J Psychiatr 2018;34:21-30.

$21 \mathrm{Li} \mathrm{J,} \mathrm{Li} \mathrm{J,} \mathrm{Thornicroft} \mathrm{G,} \mathrm{et} \mathrm{al.} \mathrm{Training} \mathrm{community} \mathrm{mental} \mathrm{health} \mathrm{staff} \mathrm{in} \mathrm{Guangzhou,}$ China: evaluation of the effect of a new training model. BMC Psychiatry 2015;15.

22 Maulik PK, Kallakuri S, Devarapalli S, et al. Increasing use of mental health services in remote areas using mobile technology: a pre-post evaluation of the smart mental health project in rural India. J Glob Health 2017;7:010408.

23 Iheanacho T, Marienfeld C, Stefanovics E, et al. Attitudes toward mental illness and changes associated with a brief educational intervention for medical and nursing students in Nigeria. Acad Psychiatry 2014;38:320-4.
24 Praharso NF, Pols H, Tiliopoulos N. Mental health literacy of Indonesian health practitioners and implications for mental health system development. Asian $J$ Psychiatr 2020;54:102168.

25 Mutiso VN, Pike K, Musyimi CW, et al. Feasibility of who mhGAP-intervention guide in reducing experienced discrimination in people with mental disorders: a pilot study in a rural Kenyan setting. Epidemiol Psychiatr Sci 2019;28:156-67.

26 Hailemichael Y, Hailemariam D, Tirfessa K, et al. Catastrophic out-of-pocket payments for households of people with severe mental disorder: a comparative study in rural Ethiopia. Int J Ment Health Syst 2019;13:39.

27 Chisholm D, Garman E, Breuer E, et al. Health service costs and their association with functional impairment among adults receiving integrated mental health care in five low- and middle-income countries: the prime cohort study. Health Policy Plan 2020;35:567-76

28 Charlson FJ, Diminic S, Lund C, et al. Mental and substance use disorders in subSaharan Africa: predictions of epidemiological changes and mental health workforce requirements for the next 40 years. PLoS One 2014;9:e110208.

29 Charlson FJ, Diminic S, Whiteford HA. The rising tide of mental disorders in the Pacific region: forecasts of disease burden and service requirements from 2010 to 2050. Asia Pac Policy Stud 2015;2:280-92.

30 Charlson FJ, Lee YY, Diminic S, et al. Applications of the epidemiological modelling outputs for targeted mental health planning in conflict-affected populations: the Syria case-study. Glob Ment Health 2016;3:e8.

31 Catalao R, Eshetu T, Tsigebrhan R, et al. Implementing integrated services for people with epilepsy in primary care in Ethiopia: a qualitative study. BMC Health Serv Res 2018;18:372.

32 Breuer E, Subba P, Luitel N, et al. Using qualitative comparative analysis and theory of change to unravel the effects of a mental health intervention on service utilisation in Nepal. BMJ Glob Health 2018;3:e001023.

33 Spagnolo J, Champagne F, Leduc N, et al. "We find what we look for, and we look for what we know": factors interacting with a mental health training program to influence its expected outcomes in Tunisia. BMC Public Health 2018;18:1398.

34 Kerbage H, Marranconi F, Chamoun Y, et al. Mental health services for Syrian refugees in Lebanon: perceptions and experiences of professionals and refugees. Qual Health Res 2020;30:849-64.

35 Miguel-Esponda G, Bohm-Levine N, Rodríguez-Cuevas FG, et al. Implementation process and outcomes of a mental health programme integrated in primary care clinics in rural Mexico: a mixed-methods study. Int J Ment Health Syst 2020;14:21.

36 Miguel Esponda G. What enables and hinders implementation? A mixed-methods case study of a mental health programme implemented in primary care clinics in rural Mexico: London School of Hygiene \& Tropical Medicine, 2020.

37 Hailemariam M, Fekadu A, Medhin G, et al. Equitable access to mental healthcare integrated in primary care for people with severe mental disorders in rural Ethiopia: a community-based cross-sectional study. Int J Ment Health Syst 2019;13:78

38 Musyimi CW, Mutiso VN, Ndetei DM, et al. Mental health treatment in Kenya: tasksharing challenges and opportunities among informal health providers. Int J Ment Health Syst 2017;11:45

39 Luitel NP, Breuer E, Adhikari A, et al. Process evaluation of a district mental healthcare plan in Nepal: a mixed-methods case study. BJPsych Open 2020;6:e77.

40 Spagnolo J, Champagne F, Leduc N, et al. A program to further integrate mental health into primary care: lessons learned from a pilot trial in Tunisia. J Glob Health Rep 2019;3.

41 Chaulagain A, Pacione L, Abdulmalik J, et al. Who mental health gap action programme intervention guide (mhGAP-IG): the first pre-service training study. Int $\rfloor$ Ment Health Syst 2020;14:47.

42 Diez-Canseco F, Toyama M, Ipince A, et al. Integration of a technology-based menta health screening program into routine practices of primary health care services in Peru (the Allillanchu project): development and implementation. J Med Internet Res 2018;20:e100

43 Rai S, Gurung D, Kaiser BN, et al. A service user co-facilitated intervention to reduce mental illness stigma among primary healthcare workers: utilizing perspectives of family members and caregivers. Fam Syst Health 2018;36:198-209

44 Hughes P, Thomson S. mhGAP - the global scenario. Prog Neurol Psychiatry 2019;23:4-6.

45 Echeverri C, Le Roy J, Worku B, et al. Mental health capacity building in refugee primary health care settings in sub-Saharan Africa: impact, challenges and gaps. Glob Ment Health 2018;5. 